\title{
The NEUF-DIX Space Project - Non-EquilibriUm Fluctuations during DIffusion in compleX liquids
}

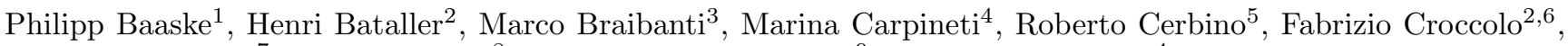 \\ Aleksandar Donev ${ }^{7}$, Werner Köhler ${ }^{8}$, José M. Ortiz de Zárate ${ }^{9}$, and Alberto Vailati ${ }^{4}$ \\ 1 Nanotemper Technologies GmbH, Munich, Germany \\ 2 Laboratoire des Fluides Complexes et leurs Réservoirs - UMR5150, Université de Pau et des Pays de l'Adour, F-64600 Anglet, \\ France \\ 3 ESA-Estec, Noordwijk The Netherlands \\ 4 Dipartimento di Fisica, Università degli Studi di Milano, I-20133 Milano, Italy \\ 5 Dipartimento di Biotecnologie Mediche e Medicina Traslazionale, Università degli Studi di Milano 20090 Segrate \\ ${ }^{6}$ Centre Nationale d'Etudes Spatiales, Paris, France \\ 7 Courant Institute of Mathematical Sciences, New York University, New York, NY 10012 \\ 8 Physikalisches Institut, Universität Bayreuth, D-95440 Bayreuth, Germany \\ 9 Department of Applied Physics I, Universidad Complutense, ES-28040 Madrid, Spain
}

Received: date / Revised version: date

\begin{abstract}
Diffusion and thermal diffusion processes in a liquid mixture are accompanied by long-range non-equilibrium fluctuations, whose amplitude is orders of magnitude larger than that of equilibrium fluctuations. The mean square amplitude of the non-equilibrium fluctuations presents a scale-free power law behavior $q^{-4}$ as a function of the wave vector $q$, but the divergence of the amplitude of the fluctuations at small wave vectors is prevented by the presence of gravity. In microgravity conditions the non-equilibrium fluctuations are fully developed and span all the available length scales up to the macroscopic size of the systems in the direction parallel to the applied gradient. Available theoretical models are based on linearized hydrodynamics and provide an adequate description of the statics and dynamics of the fluctuations in the presence of small temperature/concentration gradients and under stationary or quasi-stationary conditions. We describe a project aimed at the investigation of Non-EquilibriUm Fluctuations during DIffusion in compleX liquids (NEUF-DIX). The focus of the project is on the investigation of the non-equilibrium fluctuations in complex liquids, trying to tackle several challenging problems that emerged during the latest years, such as the theoretical predictions of Casimir-like forces induced by non-equilibrium fluctuations; the understanding of the non-equilibrium fluctuations in multi-component mixtures including a polymer, both in relation to the transport coefficients and to their behavior close to a glass transition; the understanding of the non-equilibrium fluctuations in concentrated colloidal suspensions, a problem closely related with the detection of Casimir forces; and the investigation of the onset of fluctuations during transient diffusion. We envision to parallel these experiments with state of the art multi-scale simulations.
\end{abstract}

PACS. XX.XX.XX No PACS code given

\section{Introduction}

A binary liquid mixture at equilibrium undergoes local concentration fluctuations determined by the thermal agitation of the molecules. Under these conditions the average concentration is a global variable (it does not depend on space or time), while the fluctuations are local. A great deal of attention has been focused on that simpler case for many decades [1-4]. Nowadays, equilibrium fluctuations are understood as a sort of white noise, i.e. fluctuations of all wavelengths have similar intensity, and

Send offprint requests to: alberto.vailati@unimi.it

fabrizio.croccolo@univ-pau.fr they are responsible for phenomena like scattering of light by a homogeneous fluid. Things change abruptly if a fluid comes close to an equilibrium critical point. In the vicinity of a second order phase transition, the fluctuation spectrum gets coloured and fluctuations start to become long ranged. Their intensity is typically larger for small wave vectors and actually they cannot be considered anymore a local property of the fluid. A large amount of research was performed in this area in the 1970s/1980s [5] but many interesting issues remain still open. For instance, it was known from long time [6] that long-ranged equilibrium fluctuations close to a critical point cause a very interesting phenomenon referred to as the critical Casimir effect. 
However, its existence has been experimentally verified only quite recently [7].

During the past twenty years, however, the interest of science moved towards the complex phenomenology of non - equilibrium fluctuations (NEFs), beginning with their first theoretical prediction $[8,9]$ for simple liquids under the action of a temperature gradient, and the recognition of their important role in all diffusive processes [4]. Linearized fluctuating hydrodynamics provides a satisfactory description of NEFs in binary liquid mixtures [10,11], that found experimental confirmation in several experiments, both on Earth [12-19] and in microgravity [20-22]. However, theory and experiments refer only to ideal conditions as for example stationary states, small gradients, and diluted systems. In recent years a number of theories [23-28] and some preliminary experiments [29-31] have indicated the importance of a deeper understanding of NEFs in more general cases.

In this paper we will present a project to investigate Non-EquilibriUm Fluctuations during DIffusion in compleX liquids (NEUF-DIX Project) in the absence of gravity. The aim is to perform experiments in conditions that cannot be easily tackled by theoretical models, such as transient diffusion, concentrated samples, multi-component mixtures and large gradients. The focus of the project is on the investigation of non-equilibrium fluctuations in complex liquids, because of the rich phenomenology that can be attained by tuning the interactions in such systems. Since gravity quenches long-wavelength non-equilibrium fluctuations, we stress the potential interest in performing this kind of experiments under microgravity conditions in order to fully exploit the scale-free behavior of the fluctuations.

The goal of the project is to deal with several challenging problems that emerged during the latest years, such as:

i) the theoretical prediction of Casimir-like forces induced by non-equilibrium fluctuations [23, 24, 26, 27];

ii) the understanding of the non-equilibrium fluctuations in multi-component mixtures, in relation to the transport coefficients [32,33];

iii) the understanding of the non-equilibrium fluctuations in polymer solutions, in relation to their behavior close to a glass transition [34,35];

iv) the understanding of the non-equilibrium fluctuations in concentrated colloidal suspensions [36,37,31], a problem closely related with the detection of Casimir forces;

v) the investigation of the onset of fluctuations during transient diffusion [21].

These experiments will be accompanied by state of the art multi-scale simulations $[38,39]$.

We propose to utilize a single versatile shadowgraph apparatus similar to the one built for the GRADFLEXmixture experiment, flown successfully in 2007 on-board the Russian satellite FOTON M3 [40,20,41,22]. This instrument is able to acquire images of refractive index fluctuations in a liquid sample subjected to a thermal stress. In fact, imposing an external temperature gradient is the easiest and technically simpler way of maintaining a nonequilibrium steady state in a liquid, although in many instances on ground, this is not possible due to convection.

In the next paragraphs we review the present understandings in the field of NEFs, and we report the most interesting recent theoretical and experimental results, enlightening the open questions and the experimental needs. We will briefly outline how the NEUF-DIX project aims at reaching a fundamental understanding of NEFs, and bridge the gap towards practical applications through the development of diagnostic tools aimed at the measurement of thermophysical properties from non-equilibrium fluctuations.

\section{Non equilibrium fluctuations}

In the presence of a macroscopic gradient (of concentration or temperature), the thermodynamic variables exhibit non equilibrium fluctuations that are drastically different from equilibrium ones [8-11,4]. First, the intensity of NEFs is strongly enhanced compared with the equilibrium ones. This enhancement can be as large as several orders of magnitude, depending on the accessible wave numbers and is only affected by the gravity force $[42,16$, $43]$ and by the finite size of the sample $[44,20,30,25]$. The strong influence of gravity is apparent from Fig. 1, comparing the fluctuations on Earth and in Space during the diffusion of a polymer solution of polystyrene in toluene [20]. A second important feature of non-equilibrium fluctuations is that they are always long-ranged, whatever the distance from critical conditions.

The statistical analysis of non-equilibrium fluctuations shows that their mean square amplitude exhibits a $q^{-4}$ power law behavior, while at small wave-vectors gravity makes it saturate at a constant value $[4,45]$ :

$$
\left\langle|\delta c(q)|^{2}\right\rangle_{\mathrm{NE}}=\left\langle|\delta c|^{2}\right\rangle_{\mathrm{EQ}} \frac{\nabla c}{\nabla c_{g}} \frac{1}{1+\left(q / q_{R O}\right)^{4}}
$$

Here $\left\langle|\delta c|^{2}\right\rangle_{\mathrm{EQ}}$ is the mean square amplitude of equilibrium concentration fluctuations, $c$ is the concentration of the mixture, $\nabla c_{g}$ is the concentration gradient determined by barodiffusion on Earth, and $q_{R O}$ is the rolloff wave-vector marking the transition between the two regimes:

$$
q_{R O}=\left(\frac{\beta g \nabla c}{\nu D}\right)^{\frac{1}{4}}
$$

where $g$ is the acceleration of gravity, $\nu$ the kinematic viscosity, $D$ the diffusion coefficient and $\beta$ the solutal expansion coefficient. In the absence of gravity the only physical effect preventing the divergence of the amplitude of fluctuations at small wave-vectors is the finite size of the sample. The relaxation of the fluctuations occurs by diffusion at large wave-vectors, while it is determined by 

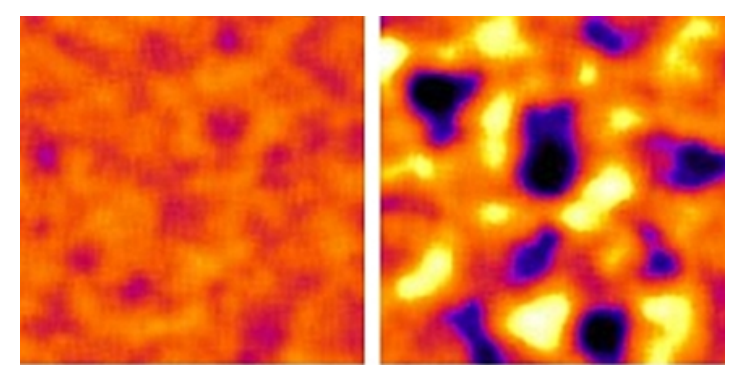

Fig. 1. False color maps of non-equilibrium concentration fluctuations during a diffusion process taking place on Earth (left) and in Space (right). The side of each image is about $4 \mathrm{~mm}$ in real space. Image courtesy of ESA/GRADFLEX Team [20]

buoyancy at smaller wave-vectors. The corresponding relaxation time also exhibits a crossover in correspondence to $q_{R O}$ :

$$
\tau(q)=\frac{1}{D q^{2}\left[1+\left(\frac{q_{R O}}{q}\right)^{4}\right]} .
$$

A true understanding of the physics behind nonequilibrium fluctuations has only gradually developed in the last couple of decades. NEFs are closely related to the transport properties of the fluid $[46,32,47,48]$. This is why from the analysis of NEFs one can determine simultaneously all transport coefficients $[32,49]$. These properties control the physico-chemical processes taking place inside the system and, hence, the observation of NEFs is particularly well-suited to probe the competition between the different mechanisms (diffusion, buoyancy, confinement...) contributing to the dynamics of the system [30]. In this sense one can think at nonequilibrium fluctuations as a probe to investigate the fluid behavior. In a single shot measurement one can obtain a wealth of information about the system. This is particularly true for complex fluids, where additional features and physical mechanisms may be present, such as particles of different size, colloidal interactions, glass transitions, and so on. Non-equilibrium fluctuations are also the seed of convective motions in the presence of gravity [43]. Investigating them in microgravity can thus unveil the mechanism behind the onset of convection without the masking effect of convection itself.

\section{The NEUF-DIX project}

The aim of the NEUF-DIX project is the investigation of non-equilibrium fluctuations associated to diffusion in complex liquids. The objectives of the project involve both the fundamental understanding of non-equilibrium fluctuations and the development of diagnostic tools based on the fluctuations (Fig. 2). So far, theoretical models able to describe the properties of non-equilibrium fluctuations have been developed only for ideal conditions like steady states, small gradients, and high dilutions [4]. Several experiments have shown the desirability of a deeper understanding of non-equilibrium fluctuations under non-ideal conditions, such as large concentrations, large gradients and time dependent processes $[34,37,21,31]$. The available theoretical models rely mostly on linearized hydrodynamics, but several recent theoretical works have shown the importance of second order terms, which give rise to nonlinear effects such as the non-equilibrium Casimir Forces $[23,24,26,27,6]$. Beyond their relevance in several natural and technological processes, complex liquids represent the ideal system to investigate non-equilibrium fluctuations, due to the opportunity of tuning the microscopic size of the system and the range of the interaction, together with the typical diffusive timescales $[17,18,36,37$, $20,21,31$. One of best tools to investigate complex liquids is represented by light scattering. Recently, several near field scattering techniques have been developed [50-53], which represent rugged and reliable alternatives to more traditional far-field light scattering techniques [54]. These near field techniques are able to operate at the small wave vectors required for the investigation of non-equilibrium fluctuations and have been recently used effectively in the GRADFLEX [40,20-22] and SODI-COLLOID [55,56] experiments performed under microgravity conditions.

The fact that the amplitude and relaxation time of non-equilibrium fluctuations depend on the Soret and diffusion coefficient has recently suggested the opportunity of using the fluctuations to achieve a rapid assessment of the transport coefficients of a multicomponent liquid mixture $[32,57,22]$. Therefore, a further goal of the NEUF-DIX project is the development and refinement of diagnostic tools based on non-equilibrium fluctuations to characterize the thermo-physical properties of complex liquid mixtures.

The NEUF-DIX project is strongly based on the important results coming from recent experiments and theoretical models, which fit the general concept of project: non-equilibrium concentration fluctuations in complex liquids under non-ideal conditions. These recent works present several aspects that need to be further investigated and that might find an answer through ad hoc comparative experiments. Due to the strong amplification or quenching effect determined by gravity on long wavelength fluctuations $[42,15,16,45,18,19,43,30]$, these experiments would greatly benefit from microgravity conditions.

\subsection{Casimir forces in equilibrium and out of equilibrium}

When fluctuations are spatially long-ranged their intensity is affected by the presence of boundaries. Likewise, the presence of such long-ranged fluctuations affects the boundaries, inducing forces on them. These effects are generically known as Casimir forces, because they were originally described by Casimir for fluctuations in the electromagnetic field [58]. When a fluid or a fluid mixture are in global thermodynamic equilibrium, fluctuations are only long-ranged in the close vicinity of the respective critical points. Hence, since the beginning of the studies on critical phenomena, it was predicted the existence of critical Casimir forces when a fluid is close to an equilibrium critical point [6]. However, fluctuation-induced forces are 


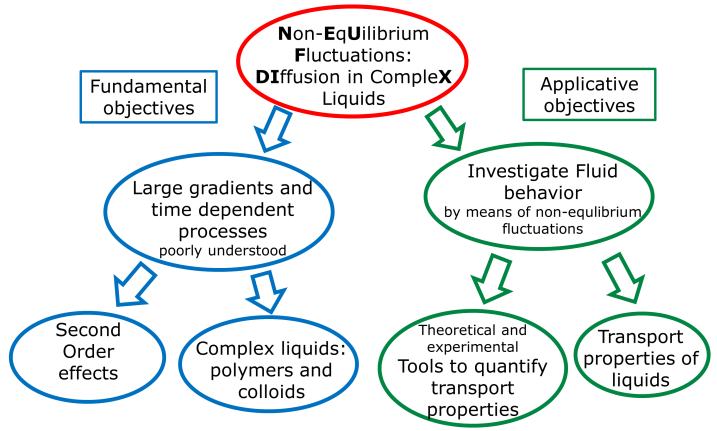

Fig. 2. Overall conceptual scheme of the Neuf-dix space project. The project aims at investigating both the fundamental and applicative features of non-equilibrium fluctuations during diffusion processes occurring under non-ideal conditions

quite feeble and it took a long time to experimentally verify, beyond any doubt, the existence of these fluctuationinduced forces $[7,59]$. The experimental study of equilibrium critical Casimir forces has expanded notably in latter years, including space research [55] where have been used to tune colloid aggregation in the SODI-COLLOID experiment, without studying the fluctuations themselves.

In parallel to these developments in equilibrium fluctuations, it has by now been well established that thermal fluctuations in fluids in non-equilibrium steady states are anomalously large and very long ranged $[8,9,4]$. The most studied case is a quiescent binary mixture in which the presence of a uniform temperature gradient induces a steady concentration gradient $\nabla c$ through the Soret effect. The intensity of NE fluctuations is given in that case by Eq. 1 that, in the absence of gravity, diverges as $q^{-4}$ when $q \rightarrow 0$. Correspondingly, in real space, the correlation length encompasses the whole spatial extension of the system [60]. Note that this is a generic result, not limited to the close vicinity of a critical point as is the case for equilibrium fluctuations.

As a consequence of this long-ranged nature of nonequilibrium fluctuations, recent theoretical research predicted the existence of a novel, yet unobserved, nonequilibrium Casimir effect that should be much more intense than the equilibrium critical one $[23,24]$. A detailed theoretical evaluation of this novel NE Casimir force is complicated by the non-existence of an out of equilibrium free energy, and by the necessity of accounting for realistic boundary conditions. The first difficulty is overcome by evaluating a fluctuation-induced density profile by expanding up to second order in the fluctuations, and later computing a Casimir pressure by using mass conservation [27], the final result being proportional to the mean-square NE fluctuations $[23,24]$. Incorporation of boundary conditions implies modifications to autocorrelations like those of Eq. 1, allowing them to be inverted to real space and to compute profiles of mean-square fluctuations in concentration or temperature meaningfully. As an example of these calculations, Fig. 3 shows the real-space mean-square NE concentration fluctuations, corresponding to Eq. 1 in microgravity conditions, once realistic boundary conditions are

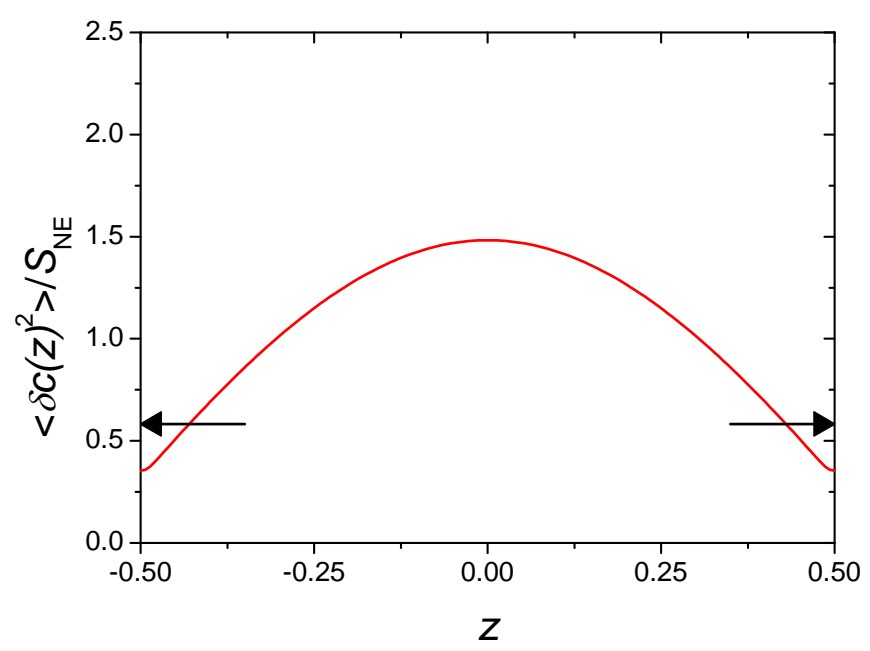

Fig. 3. Normalized mean-square value of the concentration fluctuations as a function of the (dimensionless) distance to the bounding plates $z \in[-0.5,0.5]$. The arrows indicate the average value in the layer, from which the corresponding NE Casimir pressure is evaluated.

implemented [25]. As explained above, the corresponding NE Casimir pressure is evaluated from the average value in the layer, indicated by arrows in the same Fig. 3 .

It is obvious that a key open scientific question is the experimental verification of the existence and intensity of this novel non-equilibrium Casimir effect that is so far unavailable. The space shadowgraph apparatus we envision is ideal for performing such an analysis. The most likely method for detecting the presence of fluctuation-induced forces would be by using colloidal particles as probes. In this respect, microgravity conditions are essential to minimize gravitational sedimentation, a mechanism that competes with (and masks) the non-equilibrium Casimir force.

We finalize this section by noting that it seems reasonable, as a first step towards the experimental verification of NE Casimir forces, to complete previous studies of critical Casimir forces in microgravity [55], focusing this time on the investigation of these equilibrium fluctuationinduced forces rather than on their effects.

\subsection{Complex fluids}

As a consequence of a large amount of theoretical and experimental work, many aspects of the behaviour of nonequilibrium fluctuations in binary liquid mixtures are currently well understood [4]. Most systems encountered in nature and technology are, however, truly multicomponent and/or contain a significant number of constituents of different molecular weight and size. Examples of these complex fluids are crude oil reservoirs, solutions of electrolytes and polyelectrolytes, solutions of polydisperse polymers, stabilized colloidal dispersions, and biological systems - to mention only a few. The experimental investigation of NEF in complex fluids has been rather limited and focused mostly onto dilute polymer solutions $[17$, 
20-22] and dilute colloidal suspensions [36,37]. Only very recently experimentalists became interested in dense colloidal suspensions [31], a promising experimental field, almost unexplored also from the theoretical point of view. In fact, the only available theory for concentration NEF in a dense colloid was published in 1994 by Schmitz [61] and much can be done in this respect with new approaches such as for instance Dynamic Density Functional Theory [38]. More recent is also the interest for ternary binary mixtures, driven and inspired by the international DCMIX project of ESA and Roscosmos [62,63]. Since both the experimental and theoretical difficulties increase considerably with the number of constituents, ternary systems are a natural choice for the next step beyond binaries: they already show characteristic features of truly multicomponent systems, such as cross diffusion, diffusion barriers or osmotic diffusion. On the other hand, they are still accessible in well-designed experiments and the number of independent diffusion coefficients stays manageable, albeit it increases from one to foursix $\mathrm{n}$ compared to binaries [64-66]. The investigation of ternary mixtures including a polymer is also very interesting for large polymer concentration close to the glass transition, where the effect of the entanglement and of the slowing down of the dynamics is expected to severely affect the properties of nonequilibrium fluctuations.

\subsubsection{Non-equilibrium fluctuations in a ternary mixture including a polymer}

The first empirical investigation on ternary mixtures in microgravity conditions has been provided by the experiments performed during the SCCO mission on-board the Russian FOTON M3 in 2007 [67-70,49] and the DCMIX \#1 campaign on-board the ISS in 2011 [62]. One of the outputs of these first experiments was that obtaining all the values of the mass diffusion coefficient matrix is quite difficult for mixtures of three similar molecules due to the fact that the two eigenvalues of the matrix are quite similar. Under these conditions it is thus difficult to separate the two eigenvalues and, in turn, almost impossible to get the cross-diffusion terms, i.e. the off-diagonal coefficients. Consequently, one ideal system for studying ternary mixtures is a mixture including at least one component with rather different molecular weight, like a mixture of Polystyrene (PS) dissolved in a binary mixture as a solvent, like the toluene/n-hexane one. In this case the two eigenvalues of the mass diffusion coefficient matrix are well separated and they can be quite well distinguished. For non-equilibrium fluctuations in a ternary mixture theory is currently available only for microgravity [33]. A further extension of the theory to the presence of gravity will be published on another paper on this same issue [71]. The theory will also need including the effect of confinement, even if analytical solutions cannot be obtained as in the case of binary mixtures [30].

One sample of PS/toluene/n-hexane has been selected for the future DCMIX \#4 mission. As stated, the choice of

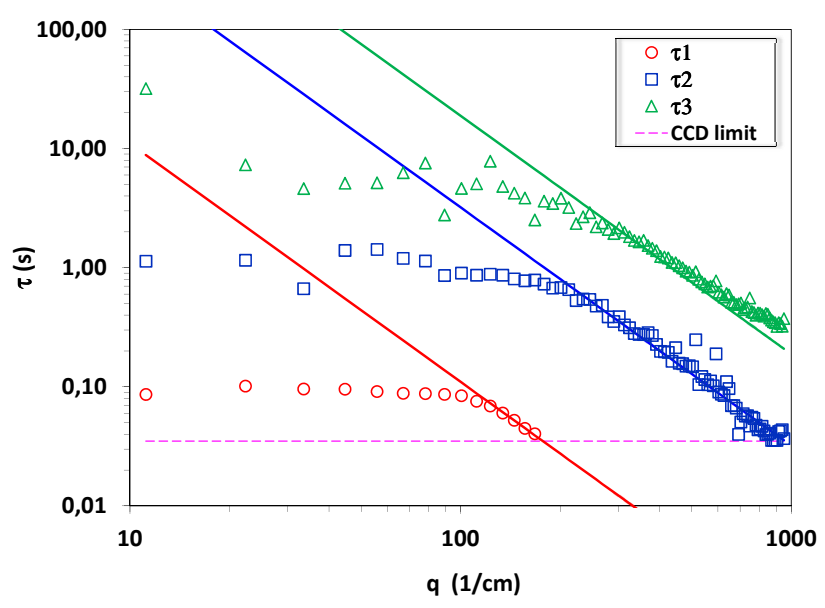

Fig. 4. Experimental decay times of NEFs in a ternary mixture of Polystyrene-toluene-hexane stressed by a temperature gradient. Three distinct time decays are clearly observable. Here $\tau_{1}$ corresponds to the fastest mode, a thermal one; $\tau_{2}$ to the intermediate one, the concentration mode for the two liquid components; and $\tau_{3}$ corresponds to the slowest mode, the concentration mode for the Polystyrene in the binary solvent. Details of this preliminary measurement are provided in another paper on this same issue. Dashed lines represent theoretical relaxation times.

a mixture of a polymer plus two molecular liquids is dictated by the need of having more distinguishable (more different) eigenvalues of the mass diffusion matrix (see Fig.4). For the NEUF-DIX project we plan to take advantage of the recording and storage of large data-sets to perform an improved statistical analysis (Bayesian inference, see Sect. 3.2.3). The combination with a Monte Carlo model based on computational fluctuating hydrodynamics [39] will allow extracting a full probability distribution of the transport coefficients of the mixture, instead of just error bars. This kind of analysis maximizes the use of experimental information without throwing away data by replacing high-dimensional data sets with fitted curves. Moreover the SODI diagnostics used for DCMIX only allows observing the concentration profiles inside the fluid slab, therefore detecting only the mass diffusion and Soret coefficients of the ternary mixture. By investigating NEFs we have demonstrated to have direct access to all the physical phenomena involved in the diffusive processes (mass diffusion, Soret, thermal diffusivity coefficients and viscosity $[32,49])$, therefore we will get more insight in the diffusion and thermodiffusion processes in a ternary system. Microgravity conditions are essential for the measurement of transport coefficients to suppress spurious effects generated by convective motions induced by temperature and concentration gradients on Earth. 


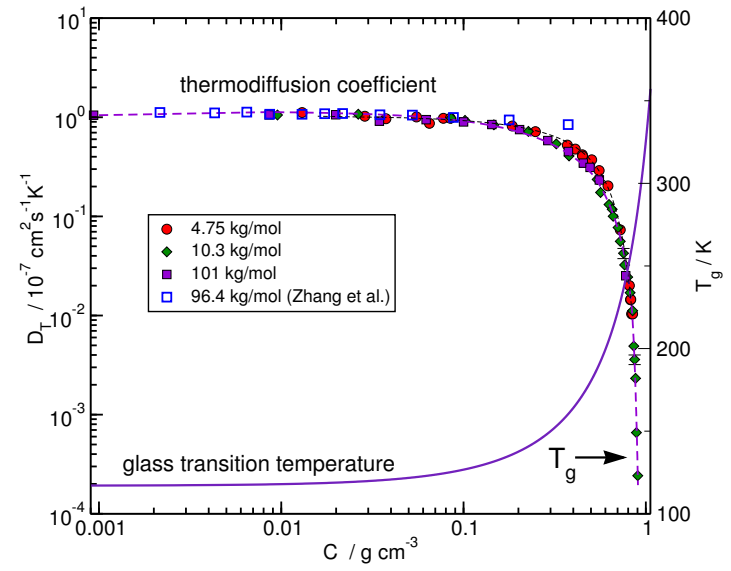

Fig. 5. Thermodiffusion coefficient $D_{T}$ and glass transition temperature $T_{g}$ as a function of polymer concentration for polystyrene in toluene according to Ref. [72]. The legends give the molar mass of the polymer. Ther literature data of Zhang et al. are from Ref. [73].

\subsubsection{Glass transition in a ternary mixture including a polymer}

The viscosity of a polymer solution and the values of certain diffusion coefficients can be tuned over an extremely wide range by comparatively minor modifications, such as changes of the concentration and/or the molar mass of the polymer. The underlying mechanisms and their consequences shall briefly be discussed in the following.

In a multicomponent mixture with a high glass transition temperature difference between the individual components, the glass transition of the mixture can be approached not only along the temperature but also along one of the composition axes. In a solution of a polymer (e.g. polystyrene) in a mixed solvent (e.g. toluene and benzene), the composition of the solvent can be varied without significantly affect the local friction and the macroscopic shear viscosity. An increase of the polymer concentration and/or an increase of the chain length, however, increases the macroscopic shear viscosity due to chain entanglement already in the semidilute regime, where the microscopic friction acting on the length scale of a polymer segment still retains its dilute solution value [74]. A further increase of the polymer concentration eventually leads to an increase of the glass transition temperature $T_{g}$ and, thus, brings the system closer to the glass phase. This results in a dramatic increase of the local friction coefficient, which, in turn, enslaves and slows-down all subsequent diffusive and viscoelastic dynamics of the system.

The slowing-down when the glass transition is approache affects both the isothermal Fickian and the thermodiffusion coefficients. As already shown for a polymer in a single solvent, both $D$ and $D_{T}$ decay by many orders of magnitude at high polymer concentration (Fig. 5). However, local friction cancels out in the Soret coefficient, which nicely follows the concentration scaling predicted by the blob model $[34,35]$. This cancellation of friction resembles the situation observed in metals for the constant ra- tio between electronic thermal and electrical conductivity, known as the the Wiedeman-Franz law [75].

The slowing-down of diffusive transport by the glass transition is in sharp contrast to the critical slowing-down near a consolute critical point, where the Soret coefficient diverges whereas the thermodiffusion coefficient remains unaffected [76]. One goal of the NEUF-DIX project is to investigate how glass transition and chain entanglement affect non-equilibrium temperature and concentration fluctuations.

\subsubsection{Dense colloidal suspensions}

Ref. [31] offers an example of the possible surprises that may arise from the study of NEF in dense colloidal suspensions, where non-local and memory effects are present. The authors developed a novel sample cell, based on liquid bridging, that allowed them to study the giant concentration NE fluctuations arising when a dense aqueous colloidal suspension is let diffuse into an overlying layer of pure water under isothermal conditions. Using a thin sample layer is quite important. In fact, the time constant characterizing isothermal diffusion is of order $L^{2} / D$, where $L$ is the sample thickness and $D$ is the diffusion coefficient of the colloidal particles. Being $D$ rather small for typical colloids, the only way to keep the experimental times reasonable is to use thin samples. The newly developed cell allowed us to perform measurements with a sample thickness $L=1 \mathrm{~mm}$. The concentration fluctuations were characterized by means of Differential Dynamic Microscopy (DDM) [77,78], a near field scattering method $[54,79]$ based on a commercial microscope that was used to obtain both static and dynamic scattering information on the suspension i.e. both the amplitude and correlation time of the concentration fluctuations as a function of their wave vector $q$ and of the time $t_{d}$ elapsed from the beginning of diffusion.

While the static scattering results were found to be in good agreement with theory, the dynamics was found to be more complex than expected. In agreement with theoretical predictions, the authors found that in the wavevector range where the NE fluctuations dominate over the equilibrium fluctuations, relaxation occurred by buoyancy for small wave-vectors, while for larger wave vectors fluctuations were found to relax diffusively with a diffusion coefficient $D_{1}$ (Fig. 6). However, for even larger $q$, in the regime where equilibrium scattering is recovered, the concentration fluctuations exhibited a diffusive relaxation with diffusion coefficient $D_{2}>D_{1}$. Even more surprisdingly, a monotonic increase of $D_{1}$ (about 15\%), was observed during diffusion in contrast with a monotonic decrease of $D_{2}$ (about $17 \%$ ). The decrease of $D_{2}$ was found to be justified by the varying concentration of the sample during diffusion. By contrast, the increase of $D_{1}$ remained unexplained and open to further investigation. Observing two different diffusion constants in this kind of experiment is especially surprising because the experiments were conducted in the hydrodynamic range, a situation in which 


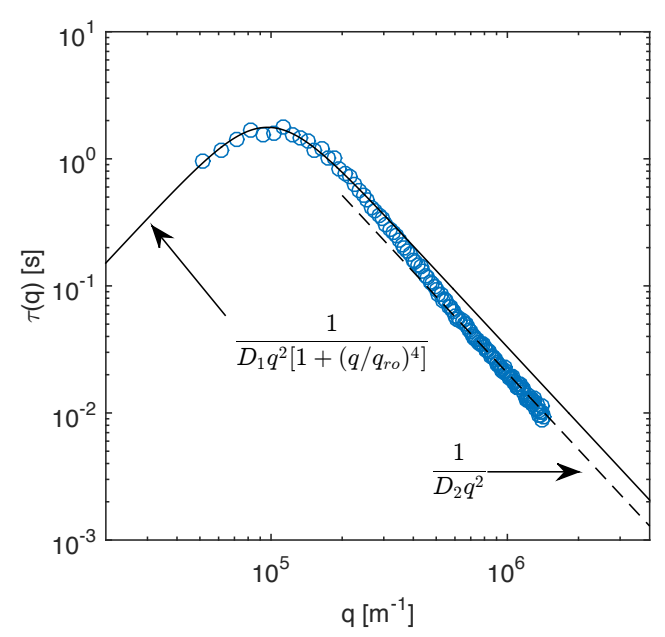

Fig. 6. Correlation time of the fast relaxation mode of concentration fluctuations in a diffusing non-equilibrium colloidal suspension as a function of the scattering wave-vector $q$, measured at approximately $700 \mathrm{~s}$ after the beginning of diffusion. The continuous line is the best fit of the low-q data with theory, which provides an estimate for the roll-off wave-vector $q_{r o}$ and for the diffusion coefficient $D_{1}$. The dashed line is a fit of the high-q data with $\tau(q)=\left(D_{2} q^{2}\right)^{-1}$, where $D_{2}>D_{1}$.

the sample should behave effectively as a molecular binary mixture.

Future experiments could take advantage of larger colloidal particles. This will allow highlighting differences with ordinary liquid mixtures in the structure and dynamics of non-equilibrium fluctuations. The use of concentrated suspensions will allow to explore regimes currently not available to analytic theoretical models and to highlight the role of interactions between the particles and possibly that of the Casimir forces induced by nonequilibrium fluctuations. On Earth, the non-equilibrium fluctuations are strongly affected by the effect of gravity, which also gives rise to the sedimentation of the particles. Under microgravity conditions the absence of the effects of buoyancy would allow achieving a better characterization of the transition between the two diffusive regimes described above. Moreover, an improve in the understanding of the non-equilibrium fluctuations in colloidal systems in space could also shed new light on the crystallization of colloids, which is known to be strongly affected by the presence of gravity [80]. Therefore, a microgravity environment is not only beneficial, but strictly needed to perform such experiments.

\subsection{Transient fluctuations in thermodiffusion separation}

So far non-equilibrium fluctuations have been investigated mostly at steady-state or during quasi-stationary processes [45], such as free diffusion $[16,18,19,31]$. A key open question is represented by the behavior of the fluctuations during the transient leading to the development of a macroscopic concentration gradient in a binary liquid mixture.

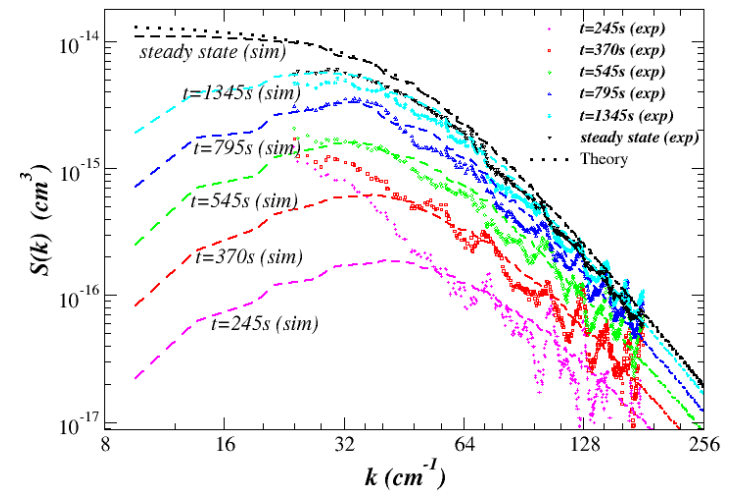

Fig. 7. Time evolution of the structure factor of nonequilibrium concentration fluctuations during the approach to steady state under microgravity conditions. Small symbols: experimental data; dashed lines: simulations; dots: exact theory [25]

Recent simulations [21] investigated the development of non-equilibrium fluctuations induced by the Soret effect in a solution of a polystyrene polymer in toluene under microgravity. The conditions mirrored those found in the GRADFLEX space experiment [20]. The sample was initially kept at a constant temperature. A temperature gradient was then suddenly applied to it to induce a mass flow through the Soret effect. This mass flow determined the gradual development of a concentration gradient inside the sample. This kind of configuration is extremely interesting, because the slow development of the macroscopic profile inside the sample allows to investigate the birth of non-equilibrium concentration fluctuations. Simulations showed that the mean squared amplitude $S(q)$ of the fluctuations as a function of wave vector exhibits the traditional power law behavior with exponent -4 at large wave vectors. However, at intermediate wave vectors $S(q)$ exhibits a peak. Quite interestingly, the time evolution of this peak is characterized by a spinodal-like growth of the fluctuations [81-83], which has been confirmed only partially by the GRADFLEX experimental data (Fig. 7) [20,21]. Future experiments will require a better statistical characterization of the transient state. This can be achieved both by iterating the experiment and by using a suspension of large colloidal particles or polymer to slow down the kinetics sufficiently to allow the investigation of the slow development of a concentration gradient induced by thermophoresis. On Earth, the presence of gravity quenches the long wave length fluctuations and inhibits completely the dynamic scaling of the fluctuations. Such inhibition would be avoided in microgravity, where the finite-size effects would acquire importance [44, $20,21,25]$.

\subsection{Numerical Simulations of Nonequilibrium Fluctuation Experiments}

The theoretical analysis of nonequilibrium thermal fluctuations uses fluctuating hydrodynamics (FHD) [4]. The 
basic idea in FHD is to add stochastic fluxes to the familiar deterministic partial differential equations (PDEs) of fluid dynamics, giving a system of stochastic PDEs (SPDEs). In order to solve these complicated SPDEs, one first linearizes the equations around the solution of the deterministic equations, and then solves the resulting linear SPDEs using (generalized) Fourier transform techniques, as illustrated on a number of relevant examples in the book [4]. This analytical approach is quite cumbersome and is only feasible if a number of additional approximations are made, many of which fail in the experiments discussed in this paper. Firstly, the deterministic state is taken to be time independent; this only applies to the study of nonequilibrium steady states (NESS) and not to transient phenomena. Secondly, the transport coefficients in the equations are assumed to be constant; this only applies to weak gradients and nearly homogeneous states. Thirdly, boundary conditions are often simplified or ignored all together because of the analytical complexity of constructing a suitable (Fourier-like) basis that respects the boundary conditions; this only works for wavelengths that are much smaller than the sample thickness. Fourthly, in order to simplify the analysis terms are often omitted, for example, inertia may be ignored or cross-coupling between temperature and concentration fluctuations may be neglected; this misses a number of relevant phenomena such as propagative (inertial) modes. Fifthly, nonlinear terms are ignored, even in the deterministic equations, and nonlinearities in the fluctuations are completely omitted in the framework of linearized fluctuating hydrodynamics; this fails at small scales $[46,84,85]$, and close to NE transitions like the convection threshold.

An alternative approach that can, in principle, remove all five approximations discussed above, is to use computer simulations to solve the equations of FHD with realistic boundary conditions, transient flows, state-dependent coefficients, large gradients, and including all nonlinear terms. Over the past decade, there has been a concerted effort to develop the required algorithms and computer codes and to apply them to the analysis of recent experiments. Developing numerical solvers for the SPDEs of FHD consists of two steps: discretizing the equations in space by using a spatial grid of cells to obtain a system of SPDEs, best done using finite volume methods [86$88]$, and then discretizing the resulting equations in time [87-89]. One of the key benefits of discretizing the original nonlinear SPDEs is that, if the fluctuations are small (cells are large and contain many molecules), the code will perform the linearization around the deterministic solution automatically without requiring any extra work [89]. Similarly, if the fluctuations are not small (cells are small and contain few molecules), the renormalization of the deterministic equations by the fluctuations will automatically be done by the code [85].

Simulations of experiments closely related to the ones described in this paper have already been carried out with notable success. The earliest work focused on the giant concentration fluctuations measured at steady state [87, 90] and during the transient [21] of the GRADFLEX ex- periment. This confirmed the applicability of FHD as a model of transient giant fluctuations, a test not possible before due to the difficulty of analytical calculations. Further work regarded fluctuations in the highly heterogeneous diffusive mixing of water and glycerol [91], the effect of confinement on the dynamics of fluctuations in a binary mixture [30], or the impact of giant fluctuations on the initial development of a doubly-diffusive gravitational instability in a ternary mixture $[92,93]$. The computational algorithms have further been extended to study giant nonequilibrium fluctuations in the presence of surface tension [94], chemical reactions [95], as well as electrostatic effects for electrolyte solutions [96]; the predictions made by the simulations have yet to be confirmed experimentally.

Computer simulations have a number of advantages as described above, however, it should be noted that they have a number of shortcomings as well. The most important one is the limitation on the range of space and time scales that can be covered due to finite computational resources, especially in three-dimensional simulations and in cases where several distinct physical processes operate at different scales. Solving the FHD equations numerically therefore requires the development of multiscale techniques, which is nontrivial in the presence of fluctuations $[89,85]$. The second limitation is that, the computer algorithms simulate a stochastic process and therefore a Monte Carlo algorithm is required to average over the fluctuations and obtain ensemble averages; this requires very long runs to obtain a large number of samples and reduce the statistical error bars in the computed averages.

The fact that the simulations generate samples of the stochastic process that is expected to mimic the real experiments is also an advantage of computer simulations, that has unfortunately not been exploited up to now. Namely, one can incorporate in the simulations a model of all of the sources of noise in the experiments, including intrinsic (thermal) fluctuations, measurement errors, external noise, etc. Once this is done, the simulations become a complete model of the experiments and can be compared to the experiments directly. This is unlike the traditional method which first processes the experimental results to remove noise and average over samples, and then fit the data with theoretical predictions in order to extract parameters [32], and compare those fitted values, rather than the directly measured stochastic samples, to theory, simulations or other experimental measurements. Instead, we propose that Bayesian inference can be used with the simulations acting as a stochastic sampler of the possible experimental outcomes, combined with actual experimental measurements, in order to extract complete posterior distributions of the relevant physical parameters. This will inform the experimentalist which parameters can actually be inferred with sufficient confidence from the measurements and allow for a meaningful quantitative comparison to alternative measurements of the same physical properties. We note that such an ambitious inverse inference program requires two challenging feats: storing a lot of raw experimental data (instead of just averages), and doing a 


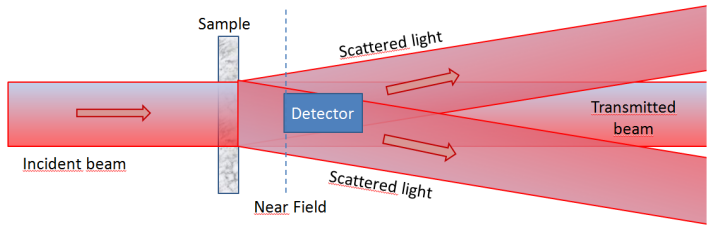

Fig. 8. Conceptual setup of near field scattering diagnostics. The interference fringes between the scattered light and the transmitted beam are collected by a detector in the near field.

large number of simulations to obtain sufficient sampling of the posterior distribution. This puts such an effort in the increasingly important and rapidly developing field of Big Data (data science).

\subsection{Experimental technique}

The ideal experimental techniques to study fluctuations in transparent dielectric samples at the mesoscopic scale are low angle scattering techniques. Local density inhomogeneities $\delta \rho(x)$ are associated to local variations of refractive index $\delta n(x)=(\partial n / \partial \rho) \delta \rho(x)$ that scatter the impinging light in the surrounding space. The intensity of the scattered light at an angle $\theta$ in the far field, is proportional to the mean square amplitude of density fluctuations $<\delta \rho(q)^{2}>$ as a function of the transferred wave vector $q=4 \pi / \lambda \sin (\theta / 2)[97,98]$. The undoubted advantage of these techniques is that they allow to make a direct measurement of the statistical properties of a sample. In fact the $\delta \rho(x)$ density distribution may be decomposed in normal modes, and in far field each mode is associated with a plane wave propagating in a well defined direction, whose intensity can be easily measured with a light detector. When density variations occur on large spatial scale, as in the case of fluctuations, the light scattered at small angles is affected by the presence of the transmitted beam. Removing the transmitted beam makes the optical alignment a very delicate task [99-101]. Besides, absolute light scattering measurements are extremely difficult, as the scattered intensity is relative to that of the incident beam, but the two are measured by different sensors.

In order to overcome these difficulties, heterodyne near field techniques are the most promising ones. The scattered pattern collected at small distances from the sample allows to recover the structure factor of the sample [50$52,102,77]$. The basic idea is that the transmitted beam and the scattered light are collected on the same detector measuring their interference pattern, as shown in Fig. 8.

In this case, the intensity measured by the sensor is given by

$$
\begin{aligned}
& I(q) \propto\left|E_{0}+E_{s}\right|^{2}=\left(E_{0}+E_{s}\right)\left(E_{0}+E_{s}\right)^{*}= \\
& \left|E_{0}\right|^{2}+2 \operatorname{Re}\left(E_{0} E_{s}^{*}\right)+\left|E_{s}\right|^{2} \simeq\left|E_{0}\right|^{2}+2 \operatorname{Re}\left(E_{0} E_{s}^{*}\right)
\end{aligned}
$$

$E_{0}$ being the amplitude of the incident field and $E_{s}$ the scattered field, with $\left|E_{s}\right|^{2}<<\left|E_{0}\right|^{2}$. The main advantages of these techniques are [54]: i) high sensitivity: signal proportional to the scattered field

ii) self referencing: absolute measurement

iii) simple optical alignment

iv) simultaneous static and dynamic measurements.

This heterodyne scheme has been the basis of various experimental techniques, both static and dynamic, such as Near Field Scattering [52], Shadowgraph [102,53], Schlieren $[103,53]$ and Differential Dynamic Microscopy [77]. All the mentioned techniques can be used either for static measurements or for dynamic ones by means of the Differential Dynamic Algorithm [104,77] possibly by taking advantage of the calculus power of modern Graphic Processor Units $[105,106]$. In particular the shadowgraph diagnostics allow to reach very small wave vectors by using an extremely simple optical setup and it's probably the most promising candidate for the integration into existing space facilities.

\section{Conclusions}

The project we propose is in-line with the strategic objectives of research in Europe. Since a better comprehension of the physics behind equilibrium fluctuations leads to several practical applications summarized above, it is foreseeable that a deeper understanding of NEFs will also pave the way to technical developments benefitting the society. In fact, the fundamental understanding of nonequilibrium fluctuations will pave the way for the development of innovative technological solutions for the rapid and non-invasive characterization of fluids out-of equilibrium, which could represent an important innovative tool in solving health and environmental issues.

\section{Acknowledgements}

We acknowledge support from the European Space Agency. We thank the GRADFLEX Team for the results in Fig. 1 and F. Giavazzi for help in preparing Fig.6. FC and HB acknowledge financial support from the Centre National d'Etudes Spatiales (CNES). JOZ thanks the Spanish 'Secretaría de Estado de Investigación' for the research grant FIS2014-58950-C2-2-P.

\section{References}

1. B. Berne, R. Pecora, Dynamic Light Scattering: With Applications to Chemistry Biology and Physics (John Wiley and Sons, New York, 1976)

2. J.P. Boon, S. Yip, Molecular Hydrodynamics (Dover, New York, 1980)

3. R.D. Mountain, J.M. Deutch, J. Chem. Phys. 20, 1103 (1969)

4. J.M. Ortiz de Zárate, J.V. Sengers, Hydrodynamic fluctuations in fluids and fluid mixtures (Elsevier, Amsterdam, 2006)

5. C. Domb, The Critical Point (Taylor \& Francis, London, 1996) 
6. M.E. Fisher, P.G. de Gennes, C. R. Acad. Sc. Paris B 287, 207 (1978)

7. C. Hertlein, L. Helden, A. Gambassi, S. Dietrich, C. Bechinger, Nature 451, 172 (2008)

8. T.R. Kirkpatrick, E.G.D. Cohen, J.R. Dorfman, Phys. Rev. A 26, 995 (1982)

9. D. Ronis, I. Procaccia, Phys. Rev. A 26, 1812 (1982)

10. B.M. Law, J.C. Nieuwoudt, Phys. Rev. A 40, 3880 (1989)

11. J.C. Nieuwoudt, B.M. Law, Phys. Rev. A 42, 2003 (1990)

12. P.N. Segrè, R.W. Gammon, J.V. Sengers, Phys. Rev. E 47, 1026 (1993)

13. W.B. Li, P.N. Segré, R.W. Gammon, J.V. Sengers, Physica A 204, 399 (1994)

14. W.B. Li, P.N. Segré, R.W. Gammon, J.V. Sengers, J. Phys.: Condens. Matter A 6, 119 (1994)

15. A. Vailati, M. Giglio, Phys. Rev. Lett. 77, 1484 (1996)

16. A. Vailati, M. Giglio, Nature 390, 262 (1997)

17. W.B. Li, K.J. Zhang, J.V. Sengers, R.W. Gammon, J.M. Ortiz de Zárate, Phys. Rev. Lett. 81, 5580 (1998)

18. D. Brogioli, A. Vailati, M. Giglio, Phys. Rev. E 61, R1 (2000)

19. F. Croccolo, D. Brogioli, A. Vailati, M. Giglio, D.S. Cannell, Phys. Rev. E 76, 041112 (2007)

20. A. Vailati, R. Cerbino, S. Mazzoni, C.J. Takacs, D.S. Cannell, M. Giglio, Nature Comm 2, 290 (2011)

21. R. Cerbino, Y. Sun, A. Donev, A. Vailati, Sci. Rep. 5, $14486(2015)$

22. F. Croccolo, C. Giraudet, H. Bataller, R. Cerbino, A. Vailati, Microgravity Sci. Technol. 28, 467 (2016)

23. T.R. Kirkpatrick, J.M. Ortiz de Zárate, J.V. Sengers, Phys. Rev. Lett. 110, 235902 (2013)

24. T.R. Kirkpatrick, J.M. Ortiz de Zárate, J.V. Sengers, Phys. Rev. Lett. 115, 035901 (2015)

25. J.M. Ortiz de Zárate, T.R. Kirkpatrick, J.V. Sengers, Eur. Phys. J. E 38, 99 (2015)

26. T.R. Kirkpatrick, J.M. Ortiz de Zárate, J.V. Sengers, Phys. Rev. E 93, 012148 (2016)

27. T.R. Kirkpatrick, J.M. Ortiz de Zárate, J.V. Sengers, Phys. Rev. E 93, 032117 (2016)

28. D. Brogioli, F. Croccolo, A. Vailati, Phys. Rev. E 94, $022142(2016)$

29. H. Bataller, C. Giraudet, F. Croccolo, J.M. Ortiz de Zárate, J. M., Microgravity Sci. Technol., accepted for publication (2016)

30. C. Giraudet, H. Bataller, Y. Sun, A. Donev, J.M. Ortiz de Zárate, F. Croccolo, Europhys. Lett. 111, 60013 (2015)

31. F. Giavazzi, G. Savorana, A. Vailati, R. Cerbino, Soft Matter 12, 6588 (2016)

32. F. Croccolo, H. Bataller, F. Scheffold, J. Chem. Phys. 137, $234202(2012)$

33. J.M. Ortiz de Zárate, C. Giraudet, H. Bataller, F. Croccolo, Eur. Phys. J. E 37, 77 (2014)

34. J. Rauch, W. Köhler, Phys. Rev. Lett. 88, 185901 (2002)

35. J. Rauch, W. Köhler, J. Phys. Chem. 119, 11977 (2003)

36. F. Croccolo, D. Brogioli, A. Vailati, M. Giglio, D.S. Cannell, Ann. N. Y. Acad. Sci. 1077, 365 (2006)

37. A. Oprisan, S. Oprisan, A. Teklu, Appl. Opt. 49, 86 (2010)

38. A. Donev, E. Vanden-Eijnden, J. Chem. Phys. 140, $234115(2014)$

39. A. Donev, A. Nonaka, A.K. Bhattacharjee, A.L. Garcia, J.B. Bell, Physics of Fluids 27, 037103 (2015)
40. A. Vailati, R. Cerbino, S. Mazzoni, M. Giglio, G. Nikolaenko, C.J. Takacs, D.S. Cannell, W.V. Meyer, A.E. Smart, Appl. Opt. 45, 2155 (2006)

41. C.J. Takacs, A. Vailati, R. Cerbino, S. Mazzoni, M. Giglio, D.S. Cannell, Phys. Rev. Lett. 106, 244502 (2011)

42. P.N. Segré, J.V. Sengers, Physica A 198, 46 (1993)

43. F. Giavazzi, A. Vailati, Phys. Rev. E 80, R015303 (2009)

44. J.M. Ortiz de Zárate, F. Peluso, J.V. Sengers, Eur. Phys. J. E 15, 319 (2004)

45. A. Vailati, M. Giglio, Phys. Rev. E 58, 4361 (1998)

46. D. Brogioli, A. Vailati, Phys. Rev. E 63, 012105 (2000)

47. A. Donev, A. Fuente, J.B. Bell, A.L. Garcia, Phys. Rev. Lett. 106, 204501 (2011)

48. A. Donev, T.G. Fai, E. Vanden-Eijnden, J. Stat. Mech. P04004, 204501 (2014)

49. I. Lizarraga, C. Giraudet, F. Croccolo, M.M. Bou-Ali, H. Bataller, Microgravity Sci. and Technol. 28, 267 (2016)

50. M. Giglio, M. Carpineti, A. Vailati, Phys. Rev. Lett. 85, 1416 (2000)

51. M. Giglio, M. Carpineti, A. Vailati, D. Brogioli, Appl. Optics 40, 4036 (2001)

52. D. Brogioli, A. Vailati, M. Giglio, Appl. Phys. Lett. 81, 4109 (2002)

53. F. Croccolo, D. Brogioli, App. Opt. 50, 3419 (2011)

54. R. Cerbino, A. Vailati, Curr. Opin. Colloid Interface Sci. 14, 416 (2009)

55. S.J. Veen, O. Antoniuk, B. Weber, M.A.C. Potenza, S. Mazzoni, P. Schall, G.H. Wegdam, Phys. Rev. Lett. 109, 248302 (2012)

56. S. Mazzoni, M.A.C. Potenza, M.D. Alaimo, S.J. Veen, M. Dielissen, E. Leussink, J.L. Dewandel, O. Minster, E. Kufner, G. Wegdam et al., Rev. Sci. Instrum. 84, 043704 (2013)

57. C. Giraudet, H. Bataller, F. Croccolo, Eur. Phys. J. E 37, 107 (2014)

58. H.B.G. Casimir, Proc. Koninklijke Nederlandse Acad. Wetenschappen B 51, 793 (1948)

59. A. Gambassi, C. Hertlein, L. Helden, C. Bechinger, S. Dietrich, Europhysics News 40, 18 (2009)

60. J.M. Ortiz de Zárate, R. Pérez Cordón, J.V. Sengers, Physica A 291, 113 (2001)

61. R. Schmitz, Physica A 206, 25 (1994)

62. M.M. Bou-Ali, A. Ahadi, D.A. de Mezquia, Q. Galand, M. Gebhardt, O. Khlybov, W. Köhler, M. Larranaga, J.C. Legros, T. Lyubimova et al., Eur. Phys. J. E 38, E0 (2015)

63. M. Gebhard, W. Köhler, Eur. Phys. J. E 38, 24 (2015)

64. W. Köhler, K.I. Morozov, J. Non-Equilib. Thermodyn. 41, 151 (2016)

65. M. Gebhardt, W. Köhler, J. Chem. Phys. 142, 084506 (2015)

66. M. Gebhardt, W. Köhler, J. Chem. Phys. 143, 164511 (2015)

67. S. Srinivasan, M.Z. Saghir, J. Chem. Phys. 131, 124508 (2009)

68. S.V. Vaerenbergh, S. Srinivasan, M.Z. Saghir, 131, 114505 (2009)

69. M. Touzet, V.L. G. Galliero, M.Z. Saghir, F. Montel, J.C. Legros, Comptes Rendus - Mécanique 339, 318 (2011)

70. G. Galliero, H. Bataller, F. Croccolo, R. Vermorel, P. a. Artola, B. Rousseau, V. Vesovic, M.M. Bou-Ali, J.M. Ortiz de Zárate, S. Xu et al., Microgravity Sci. and Technol. 28, 79 (2016) 
71. P. Martinez Pancorbo, J.M. Ortiz de Zárate, H. Bataller, F.C. and, Eur. Phys. J. E., submitted (2016)

72. J. Rauch, W. Köhler, Phys. Rev. Lett. 88, 185901 (2003)

73. K.J. Zhang, M.E. Briggs, R.W. Gammon, J.V. Sengers, J.F. Douglas, J. Chem. Phys. 111, 2270 (1999)

74. K.I. Morozov, W. Köhler, Langmuir 30, 6571 (2014)

75. R. Franz, G. Wiedemann, 165, 497 (1853)

76. W. Enge, W. Köhler, Phys. Chem. Chem. Phys. 6, 2373 (2004)

77. R. Cerbino, V. Trappe, Phys. Rev. Lett. 100, 188102 (2008)

78. F. Giavazzi, D. Brogioli, V. Trappe, T. Bellini, R. Cerbino, Phys. Rev. E 80, 031403 (2009)

79. F. Giavazzi, R. Cerbino, J. Opt. 16, 83001 (2014)

80. J. Zhu, M. Li, R. Rogers, W. Meyer, W.R. R. H. Ottewill, STS-73 Space Shuttle Crew, P. Chaikin, Nature 387, 883 (1997)

81. J.S. Huang, W.I. Goldburg, A.W. Bjierkaas, Phys. Rev. Lett. 32, 921 (1974)

82. K. Binder, D. Stauffer, Phys. Rev. Lett. 33, 1006 (1974)

83. M. Carpineti, M. Giglio, Phys. Rev. Lett. 68, 3327 (1992)

84. A. Donev, A.L. Garcia Anton de la Fuente, J.B. Bell, Phys. Rev. Lett. 106, 204501 (2011)

85. A. Donev, T.G. Fai, E. Vanden-Eijnden, Theor. Exp 2014, P04004 (2014)

86. A. Donev, E. Vanden-Eijnden, A.L. Garcia, J.B. Bell, Comm. App. Math. Comp. Sci. 5, 149 (2010)

87. F.B. Usabiaga, J.B. Bell, R. Delgado-Buscalioni, A. Donev, T.G. Fai, B.E. Griffith, C.S. Peskin, Multiscale Model. Simul. 10, 1369 (2012)

88. S. Delong, B.E. Griffith, E. Vanden-Eijnden, A. Donev, Phys. Rev. E 87, 033302 (2013)

89. S. Delong, Y. Sun, B.E. Griffith, E. Vanden-Eijnden, A. Donev, Phys. Rev. E 90, 063312 (2014)

90. A. Donev, A.J. Nonaka, Y. Sun, T.G. Fai, A.L. Garcia, J.B. Bell, Comm. App. Math. Comp. Sci. 9, 47 (2014)

91. A.J. Nonaka, Y. Sun, J.B. Bell, A. Donev, Comm. App. Math. Comp. Sci. 10, 163 (2015)

92. A. Donev, A.J. Nonaka, A.K. Bhattacharjee, A.L. Garcia, J.B. Bell, Phys. Fluids 27, 037103 (2015)

93. J. Carballido-Landeira, P.M.J. Trevelyan, C. Almarcha, A.D. Wit, Phys. Fluids 25, 024107 (2013)

94. A. Chaudhri, J.B. Bell, A.L. Garcia, A. Donev, Phys. Rev. E 90, 033014 (2014)

95. A.K. Bhattacharje, K. Balakrishnan, A.L. Garcia, J.B. Bell, A. Donev, J. Chem. Phys 142, 224107 (2015)

96. A. Chaudhri, J.B. Bell, A. Donev, J. p. Peraud, A. Nonaka, A.L. Garcia, Submitted to Phys Rev. F, ArXiv:1607.05361 (2016)

97. H.C. Van de Hulst, Light scattering by small particles (Dover, New York, 1976)

98. M. Kerker, The scattering of light (Academic, New York, 1969)

99. A.P.Y. Wong, P. Wiltzius, Rev. Sci. Instrum. 64, 2547 (1993)

100. F. Ferri, Rev. Sci. Instrum. 68, 2265 (1997)

101. L. Cipelletti, D.A. Weitz, Rev. Sci. Instr 70, 5 (1999)

102. S.P. Trainoff, D.S. Cannell, Physics of Fluids 14, 1340 (2002)

103. D. Brogioli, A. Vailati, M. Giglio, Europhys. Lett. 63, 220 (2003)

104. F. Croccolo, D. Brogioli, A. Vailati, M. Giglio, D.S. Cannell, App. Opt. 45, 2166 (2006)
105. G. Cerchiari, F. Croccolo, F. Cardinaux, F. Scheffold, Rev. Sci. Instrum. 83, 106101 (2012)

106. P.J. Lu, F. Giavazzi, T.E. Angelini, E. Zaccarelli, F. Jargstorff, A.B. Schofield, J.N. Wilking, M.B. Romanowsky, D.A. Weitz, R. Cerbino, Phys. Rev. Lett. 108, 218103 (2012) 